\title{
Lernen vom Besten
}

\section{Annette Blaudszun}

In den Werkstätten für behinderte Menschen zeichnet sich, wie in der Sozialwirtschaft generell, die Tendenz ab, die Qualität der Leistungserbringung gegenüber der Öffentlichkeit und den Kostenträgern auszuweisen. Während betriebswirtschaftlich orientierte Kennzahlen bereits seit Längerem erhoben und ausgetauscht werden, fehlten bislang Verfahren und Indikatoren zur Qualitätsbeurteilung der Leistungserbringung im zentralen Aufgabenbereich der Werkstätten, der Rehabilitation und Förderung behinderter Menschen weitgehend. Ein Projekt zur Entwicklung und Einführung eines kennzahlenbasierten Rehabilitationsvergleiches sollte diesem Mangel abhelfen.

$\mathrm{N}$ achdem in den letzten Jahren vorrangig die Implementierung von eher verfahrensorientierten Qualitätssicherungssystemen in Werkstätten für behinderte Menschen betrieben wurde, gewinnt der Aspekt der systematischen, kontinuierlichen Qualitätsförderung zunehmend an Bedeutung. Die verstärkte Auseinandersetzung um die Qualität sozialer Dienstleistungen ist vorrangig auf die Verknappung öffentlicher Mittel zurückzuführen.

Ziel der in den letzten Jahren eingeführten gesetzlichen Bestimmungen ist neben der Qualitätssicherung und Qualitätsverbesserung die Senkung der Kosten sowie eine stärkere Kontrolle über die Verwendung öffentlicher Mittel. Auch wenn die Verschränkung von Kostensenkungsund Qualitätsaspekten kontrovers diskutiert werden kann, zeichnet sich doch auch im Non-Profit-Bereich die Tendenz ab, die Qualität der Leistungserbringung im Zuge der kontinuierlichen Qualitätsverbesserung oder zum Zweck der Leistungsverein- barung mit Kostenträgern auszuweisen.

Für die Rehabilitation und Teilhabe behinderter Menschen gibt das Neunte Sozialgesetzbuch den Leistungserbringern die Sicherstellung eines Qualitätsmanagements vor und verlangt durch zielgerichtete und systematische Verfahren und Maßnahmen die Qualität der Versorgung zu gewährleisten und kontinuierlich zu verbessern. Dabei wird die Durchführung vergleichender Qualitätsanalysen als Grundlage für ein effektives Qualitätsmanagement bezeichnet (vgl. SGB IX, § 201 , 2)).

Mit den gesetzlichen Vorgaben zur Qualitätssicherung (SGB IX, § 20) sowie zur Förderung und Rehabilitation in Werkstätten für behinderte Menschen (SGB IX, § 39) besteht ein Bedarf zur Evaluation Sozialer Arbeit, der über bisher vorhandene Instrumente kaum gedeckt werden kann. Während betriebswirtschaftlich orientierte Kennzahlen bereits seit Längerem erhoben und auch zwischen Einrichtungen ausgetauscht und verglichen werden, fehlten bislang Verfahren und Indikatoren zur Qualitätsbeurteilung der Leistungserbringung

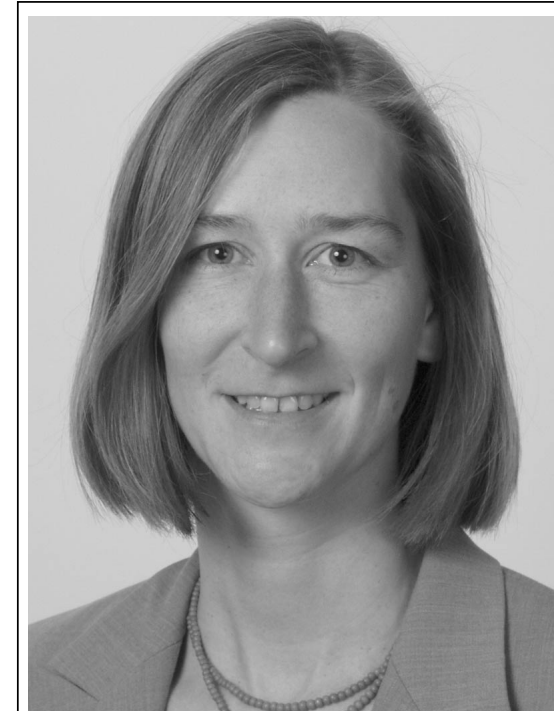

Annette Blaudszun ist Krankenschwester und Diplom-Pflegewirtin. Nach dem Studium der Pflegewissenschaft arbeitete sie mehrere Jahre in der Schweiz an der Entwicklung und Umsetzung von Outcome-Messungen für den Krankenhausbereich und an der Durchführung von verschiedenen Benchmarking-Projekten im Gesundheitswesen. Seit 2002 ist sie wissenschaftliche Mitarbeiterin am Institut für Technologie und Arbeit - ITA - in Kaiserslautern (http://www.ita-kl.de). Schwerpunkt ihrer Tätigkeit ist die Entwicklung von Qualitätsmessungen und die Initialisierung von Benchmarkingprozessen in Werkstätten für behinderte Menschen.

E-Mail:

annette.blaudszun@ita-kl.de

\section{Die beteiligten Werkstätten des DRK-Landesverbandes Nordrhein}

Gemeinnützige Werkstätten Köln GmbH (GWK) Gemeinnützige Werkstätten Neuss GmbH (GWN) Werkstatt Lebenshilfe i. Bergischen Land $\mathrm{GmbH}$ (WLH) Behinderten Werkstätten Oberberg $\mathrm{GmbH}$ (BWO) WFB Werkstätten des Kreises Mettmann $\mathrm{GmbH}$ Haus Freudenberg $\mathrm{GmbH}$ RAPS Gemeinnützige Werkstätten $\mathrm{GmbH}$ Wissenschaftliche Projektleitung Institut für Technologie und Arbeit (ITA) e. V. an der Technischen Universität Kaiserslautern www.gwk-koeln.de www.gwn-neuss.de www.wlh.de www.bwo-wiehl.de www.wfbme.de www.haus-freudenberg.de www.wfbm-raps.de 


\begin{tabular}{|c|c|}
\hline Projektschritte & \\
\hline $\begin{array}{l}\text { Projektaufbau, bedarfsorientierte Konkretisierung der } \\
\text { Projektplanung } \\
\text { Planung der einzelnen Projektschritte } \\
\text { Aufbau einer Projektorganisation in den beteiligten Werk- } \\
\text { stätten für behinderte Menschen } \\
\text { Verabschiedung einer verbindlichen Arbeits- und Vertrau- } \\
\text { ensvereinbarung }\end{array}$ & $\begin{array}{l}\text { Oktober bis } \\
\text { Dezember } 2002\end{array}$ \\
\hline $\begin{array}{l}\text { Entwicklung der Qualitätsmessungen } \\
\text { Entwicklung von Kennzahlen und Mitarbeiterbefragung } \\
\text { Entwicklung von Messinstrumenten und Entwicklung von } \\
\text { Maßnahmen zur Erreichung hoher Datenqualität } \\
\text { Aufbau einer stabilen Messorganisation innerhalb der } \\
\text { Werkstätten für behinderte Menschen }\end{array}$ & $\begin{array}{l}\text { Januar } 2003 \text { bis } \\
\text { März } 2004\end{array}$ \\
\hline $\begin{array}{l}\text { Datenerfassung und statistische Auswertung } \\
\text { Durchführung der Qualitätsmessungen durch die Werk- } \\
\text { stätten für behinderte Menschen und Datentransfer an die } \\
\text { Auswertungsstelle } \\
\text { Statistische Datenauswertung und Datenaufbereitung }\end{array}$ & $\begin{array}{l}\text { April bis } \\
\text { Oktober } 2004\end{array}$ \\
\hline $\begin{array}{l}\text { Initiierung des Benchmarkingprozesses } \\
\text { Ergebnispräsentation } \\
\text { Gemeinsame Interpretation und Analyse der Ergebnisse } \\
\text { »Lernen vom Besten« }\end{array}$ & $\begin{array}{l}\text { November } 2004 \\
\text { bis Februar } 2005\end{array}$ \\
\hline $\begin{array}{l}\text { Stabilisierung des Vorgehens und Projektabschluss } \\
\text { Überarbeitung der Qualitätsmessungen und der Messin- } \\
\text { strumente } \\
\text { Unterstützung zur eigenständigen Weiterführung der } \\
\text { Qualitätsmessungen und des Benchmarkings }\end{array}$ & $\begin{array}{l}\text { März bis } \\
\text { September } 2005\end{array}$ \\
\hline
\end{tabular}

Tabelle 2: Wie das Projekt durchgeführt wurde

\section{Beispiele für Kennzahlen}

1 Anzahl Mitarbeiter mit vollständigem, individuellem Förderplan, der nicht älter als ein Jahr ist

2 Entwicklung der Sozial- und Handlungskompetenz zwischen

Beginn und 1 Jahr nach Beginn der Berufsbildungsmaßnahme

Beginn und Ende des Berufsbildungsbereichs

3 Anzahl Praktika, die von Mitarbeitern des Arbeitsbereichs absolviert werden

4 Anzahl wahrgenommener Stunden an arbeitsbegleitenden Maßnahmen von Mitarbeitern mit Behinderungen pro Messzeitraum

5 Anzahl wahrgenommener Fortbildungsstunden durch das Fachpersonal pro Messzeitraum

Tabelle 3: Wie die Kennzahlen aussehen im zentralen Aufgabenbereich der Werkstätten für behinderte Menschen, der Rehabilitation und Förderung behinderter Menschen weitgehend.

\section{Das Projektziel}

Als Benchmarking im Non-ProfitBereich wird der systematische, datenbasierte Vergleich von Organisa-

\section{Themenblöcke der Mitarbeiterbefragung}

1 Arbeiten und Mitwirken

2 Arbeitsbegleitende Maßnahmen

3 Unterstützung und Hilfestellung

Bei Bedarf Erweiterung durch Zusatzmodule zu den Themen »Mittagessen«, »Weg zur Arbeit«, »Lohn« und »Werkstattrat« möglich

Tabelle 4: Wonach die Mitarbeitenden gefragt wurden tionen bezeichnet, der darauf abzielt, »vom Besten zu lernen « und die Qualität der eigenen Leistungserbringung kontinuierlich zu verbessern. Dies war das Ziel eines dreijährigen Forschungsprojektes, das Ende letzten Jahres erfolgreich zu Ende gegangen ist. Mit ihm sollte durch die Entwicklung einheitlicher Qualitätsmessungen und die Einführung eines systematischen Benchmarkingprozesses eine Verbesserung der für die Förderung und Rehabilitation behinderter Menschen relevanten Prozesse erfolgen. Darüber hinaus sollten den Entscheidungsträgern in Werkstätten für behinderte Menschen mehr Erkenntnisse über Ursache-Wirkungs-Zusammenhänge in den Förderungs- und Rehabilitationsprozessen zur Verfügung gestellt werden, um eine (förder-) zielgerichtete und effektive Steuerung der Werkstätten zu ermöglichen.

Die Projektidee beruht darauf, Qualitätsverbesserungen nicht im Alleingang umzusetzen, sondern sich zu einem Verbund mit anderen

Werkstätten zusammen zu schließen und durch externe Beratung unterstützen zu lassen. Initiiert wurde das Projekt durch die Werkstätten des Landesverbandes Nordrhein des Deutschen Roten Kreuzes, die wissenschaftliche Begleitung übernahm das Institut für Technologie und Arbeit e. $V$. an der Technischen Universität Kaiserslautern (siehe Tabelle 1).

\section{Der Prozess}

Um datenbasiert die Qualität von Förderung und Rehabilitation überprüfen, vergleichen und verbessern zu können, wurde ein Kennzahlensystem benötigt, welches das Spektrum der Leistungserbringung möglichst umfassend und nutzerorientiert abdeckt (Tabelle 2). Da auf solche Kennzahlen nicht zurückgegriffen werden konnte, wurde ein praxisorientiertes Verfahren gewählt, das eine enge Einbindung der Werkstätten in den Entwicklungsprozess entsprechender Kennzahlen gewährleistete. Damit war sichergestellt, dass das entstehende Kennzahlensystem sowohl den Bedürfnissen der Werkstätten entsprach als auch deren Fachkompetenz in höchstmöglichem Maß nutzte. Die Beurteilung der erbrachten Leistungen wurde dabei als $\mathrm{Zu}$ sammenhang zwischen so genannter »objektiver « Messung von Kennzah- 


\section{Inhalte der}

\section{Kollektivbeschreibungen}

1 Art und Ausprägung der Behinderung

2 Fähigkeitsanalyse

3 Bereich, in dem der Mitarbeiter mit Behinderungen eingesetzt ist

4 Soziodemographische Informationen (z. B. zu Alter, Wohnsituation, Beschäftigungsumfang)

Tabelle 5: Was die Kollektivbeschreibungen enthalten

len und dem »subjektiven « Erleben der Mitarbeiter mit Behinderungen verstanden.

Die entwickelte Qualitätsmessung gliedert sich in drei Bereiche:

- die Kennzahlen im Sinne objektiver Indikatoren für Rehabilitationsund Förderungsqualität

- die Mitarbeiterbefragung zur Abbildung des subjektiven Erlebens von Rehabilitations- und Förderungsleistungen durch die Mitarbeiter mit Behinderungen

- die Kennzahlen zur Kollektivbeschreibung zur Abbildung der Behinderungsarten, Fähigkeiten, soziodemographischen Informationen u. v. m.

Grundlage für die Entwicklung von Kennzahlen zur Abbildung von Rehabilitations- und Förderungsleistungen in Werkstätten für behinderte Menschen war eine gemeinsam erarbeitete Definition von Qualität. Ausgehend von dieser Definition leiteten sich die Kennzahlen sowohl aus den festgelegten Qualitätszielen als auch aus den Kernprozessen der Förderung und Rehabilitation ab. Zur Befragung der Mitarbeiter mit Behinderungen wurde die Definition für die Ableitung der relevanten Befragungsdimensionen herangezogen.

Um eine umfassende Beurteilung der Leistungsqualität zu gewährleisten, wurden bei der Entwicklung folgende Qualitätskomponenten berücksichtigt:

- die Entwicklung von Sozial- und Handlungskompetenz bei Mitarbeitern mit Behinderungen (= Ergebnisqualität)

- die Förderungs- und Rehabilitati- onsleistung von Werkstätten für behinderte Menschen (= Prozessqualität)

- die wesentlichen Rahmenbedingungen für hohe Prozess- und Ergebnisqualität (= Strukturqualität)

Die Umsetzung der Messung fand auf der Basis einheitlicher und zeitgleicher Selbstmessungen durch die beteiligten Werkstätten für behinderte Menschen statt. Zugrunde liegendes Ziel war dabei, den Werkstätten ein Instrument zur kontinuierlichen Qualitätsförderung zur Verfügung zu stellen, ohne langfristig auf externe Unterstützung angewiesen zu sein.

Die Entwicklung und Erprobung der Messinstrumente für das Kennzahlensystem und die Mitarbeiterbefragung erfolgten in einem mehrstufigen Verfahren. Während der Entwicklungsphase wurden diese immer wieder auch durch das Fachpersonal der beteiligten Werkstätten für behinderte
Fragen während der Messung

Beispiele werkstattexterner Maßnahmen:

- Entwicklung einheitlicher Messinstrumente

- Entwicklung von Richtlinien für die Umsetzung einzelner Messungen wie der Mitarbeiterbefragung

- Entwicklung eines Handbuchs zur Qualitätsmessung

- Entwicklung einheitlicher Tabellen zum elektronischen Datentransfer

Zur Messung der Kennzahlen wurden für jede einzelne Kennzahl so genannte Mess-Sets entwickelt, die von allen Werkstätten in dieser Form verwendet wurden. Diese Mess-Sets bestehen aus zwei Teilen:

- einem Kennzahlenblatt, das alle relevanten Informationen zur Kennzahl enthält wie die genaue Definition der Kennzahl, die Angaben

\section{»Mit einem Kennzablensystem soll das Spektrum der Leistungserbringung umfassend und nutzerorientiert abgedeckt werden "}

Menschen beispielsweise auf Eindeutigkeit der Definitionen oder leichte Handhabbarkeit überprüft.

Wesentliche Voraussetzung für die Nutzung von Qualitätsmessungen, insbesondere für das Benchmarking, ist das Erreichen einer hohen Datenqualität. Hierzu wurden - neben der sorgfältigen Entwicklung der Messinstrumente - verschiedene Maßnahmen werkstattintern wie auch werkstattextern umgesetzt.

Beispiele werkstattinterner Maßnahmen:

- Aufbau einer stabilen Messorganisation: festlegen von Aufgaben und Verantwortungsbereichen für die reibungslose Umsetzung der Messung sowie die Einhaltung der verbindlichen Richtlinien

- Einrichtung eines Mess-Controllings: überprüfen der Vorgaben zu den Messungen durch fest installierte Feedbackschlaufen

- Einrichtung einer Hotline für jede Kennzahl zur Klärung dringender zum betreffenden Kollektiv, Anga-

ben zum Messzeitpunkt, zur verantwortlichen Person innerhalb der Werkstatt, zum Datentransfer an die Auswertungsstelle usw.

- einem Datenblatt zur Erhebung der kennzahlenrelevanten Daten

Die Mitarbeiterbefragung wurde als mündliche Befragung umgesetzt. Ziel war hierbei, einen möglichst hohen Anteil des Gesamtkollektivs der Mitarbeiter mit Behinderungen zu erreichen. Auf der Basis einheitlicher Schulungen und einer verbindlichen Richtlinie zur Umsetzung der Befragung wurden die Mitarbeiter mit Behinderungen anhand eines strukturierten Interviewleitfadens durch das Fachpersonal der Werkstätten für behinderte Menschen befragt. Von der Messung ausgeschlossen wurden die Mitarbeiter mit Behinderungen, die nach Einschätzung der Gruppenleitung und des Sozialdienstes aufgrund zu geringer intellektueller und/oder kommunikativer Fähigkeiten nicht in der Lage waren, die Fragen zu beantworten. (In der vorliegenden ersten 
Mitarbeiterbefragung wurden bei einem Gesamtkollektiv von 4.089 Mitarbeitern mit Behinderungen 2.987 Interviews abgeschlossen). Die Interviews wurden durch Personen durchgeführt, die weder die betreffenden Mitarbeiter mit Behinderungen kannten noch an der Entwicklung des Interviewleitfadens beteiligt waren.

Um differenzierte Angaben zum Kollektiv zu erhalten, wurden neben der Behinderungsart und soziodemographischer Daten auch Fähigkeiten erhoben. Beurteilt wurden fünf Fähigkeitsbereiche, die ein umfassendes Bild des Mitarbeiters mit Behinderungen in Bezug auf kognitive, soziale und psychomotorische Fähigkeiten sowie hinsichtlich der Art der Arbeitsausführung ermöglichen. Diese Messung erfolgte anhand eines Erhebungsbogens, der sowohl die Definition der jeweiligen Fähigkeit als auch die fünf qualitativ formulierten Ausprägungsstufen vorgab. Eine verbindliche Richtlinie und die Durchführungen von Schulungen für das zuständige Fachpersonal stellten ein weitestgehend einheitliches Vorgehen sicher.

Die Auswertung und Aufbereitung der Daten sowie die Ergebnispräsentation übernahm das Institut für Technologie und Arbeit (ITA) in Kaiserslautern. In mehreren Benchmarking-Workshops analysierten und interpretierten die Benchmarkingpartner gemeinsam die Auswertungsergebnisse. Anhand ausgewählter Kennzahlen stellten die jeweils besten Werkstätten ihre Ergebnisse und die ihnen zugrunde liegenden Prozesse und Strukturen im Sinne von »best practices « vor und diskutierten diese in einem strukturierten und moderierten Prozess mit den übrigen beteiligten Werkstätten. Zwischenzeitlich wird das Benchmarking ohne externe Begleitung durch die beteiligten Werkstätten weitergeführt.

\section{Ergebnisse}

Den beteiligten Werkstätten stehen nach Abschluss des Projektes gut handhabbare Qualitätsmessungen zur Verfügung, die eine umfassende Beurteilung der Rehabilitations- und Förderungsleistung in Werkstätten für behinderte Menschen ermöglichen. Diese Qualitätsmessungen sind als Selbstevaluation konzipiert und gliedern sich in drei Teile (Tabelle 3 bis 5):

- ein aus 16 Kennzahlen bestehendes Kennzahlensystem, das sich aus den Kernprozessen und den Qualitätszielen der Förderung und Rehabilitation in Werkstätten für behinderte Menschen ableitet

- ein strukturierter Interviewleitfaden zur Befragung der Mitarbeiter mit Behinderungen mit dem Schwerpunkt Förderung und Rehabilitation sowie mehrerer Zusatzmodule

- Kennzahlen zur differenzierten Kollektivbeschreibung einschließlich einer Fähigkeitsanalyse

Damit steht den beteiligten Werkstätten ein Instrumentarium zur Analyse von Stärken und Verbesserungspotenzialen im zentralen

Aufgabenbereich von Werkstätten für behinderte Menschen, der Förderung und Rehabilitation, zur Verfügung.

Erstmals können Verbesserungsmaßnahmen datenbasiert umgesetzt werden, wodurch knappe Ressourcen effizient genutzt und die

Entscheidungen für umzusetzende Qualitätsverbesserungen sowohl dem Fachpersonal als auch den Mitarbeitern mit Behinderungen transparent gemacht werden können. Die weitgehend schon in Werkstätten etablierten betriebswirtschaftlichen Kennzahlen erhalten durch die Kennzahlen zur Rehabilitation und Förderung ein entsprechendes Gegengewicht.

Vor allem aber bieten einheitliche Qualitätsmessungen die Grundlage für einen systematischen Vergleich mit dem »best in class « und »best in process « und die Möglichkeit voneinander zu lernen. Dieses Lernen voneinander im Sinne eines effizienten Lernprozesses kann jedoch nur dann stattfinden, wenn Vertrauen und Offenheit sowie die Bereitschaft zur Kooperation gegeben sind. Die Vernetzung der beteiligten Werkstätten und der Wunsch zur transparenten Offenlegung der Messergebnisse innerhalb des Projektes haben dabei wesentlich zum Projekterfolg beigetragen.

\section{Ausblick}

Das Projekt war darauf ausgelegt, den beteiligten Werkstätten die notwendige Kompetenz zur Weiterführung der Qualitätsmessungen, zur
Neu- und Weiterentwicklung von Kennzahlen sowie zur selbstständigen Weiterführung des Benchmarkingprozesses zu vermitteln.

Mit dem Projektende vereinbarte das Netzwerk der sieben Werkstätten die kontinuierliche Weiterführung der Qualitätsmessungen und des Benchmarkings. Darüber hinaus soll eine Erweiterung des Benchmarkingpools durch interessierte Werkstätten für behinderte Menschen erfolgen. Werkstätten, die sich am Benchmarking beteiligen möchten, können die vorliegenden Qualitätsmessungen einschließlich der entwickelten Messinstrumente, der vereinbarten Maßnahmen zur Erreichung einer hohen Datenqualität sowie die Leistungen zur Datenauswertung und Datenpräsentation nutzen.

\section{Wo man weitere Informationen erhält}

Kontaktadresse:

Annette Blaudszun Institut für Technologie und Arbeit (ITA) e. V. Gottlieb-Daimler-Straße, Gebäude 42 67663 Kaiserslautern Telefon 0631 205-3737 Fax 06312053397 E-Mail benchmarking@ita-kl.de http://www.ita-kl.de 